\title{
How vertical relationships and external funding affect investment efficiency and timing?
}

\author{
Dimitrios Zormpas*
}

March 21, 2017

\begin{abstract}
In this paper we consider a potential investor who contemplates entering an uncertain new market under two conditions: i) a prerequisite for the project to take place is the purchase of a discrete input from an upstream firm with market power and ii) the completion of the investment is conditional on the participation of an investment partner who is willing to bear some of the investment cost receiving compensation in return.

Using the real option approach, we find that the involvement of any of the two alien agents causes the postponement of the completion of the investment and we discuss how these timing discrepancies are reflected on the value of the option to invest in the project. We next analyze the synchronous effect of outsourcing and external funding both in a non-cooperative and in a cooperative (Nash bargaining solution) game-theoretic setting and we show how the endogeneity of the sunk investment cost affects the timing and the value of the option to invest in projects characterized by uncertainty and irreversibility.

KEYWORDS: Investment analysis, Real options, Vertical relations, Nash bargaining solution. Jel Classification: C61, D92, G30.
\end{abstract}

\section{Introduction}

Innovation is an important factor for a company's success and a crucial explanation for observed differentials in performance (McGrath and Nerkar, 2004). Consequently, a fundamental problem that a firm faces has to do with the decision to invest in a new product, technology or service market. These kinds of managerial decisions are usually characterized by risky, irreversible and lumpy investments that are often beyond the resources of a single firm (Chesbrough and Schwartz, 2007) and, as a result, an investment partner willing to share the cost of betting on the success of the business plan under consideration is frequently sought after (Kogut, 1991). According to Quinn (2000), using partnerships "companies have lowered innovation costs and risks by $60 \%$ to $90 \%$, while similarly decreasing cycle time and leveraging their internal investments by tens to hundreds of times".

Investment partnerships might take the form of joint ventures, independent or corporate venture capital investments, strategic alliances or merges. Irrespective of the exact nature of the partnership, the underlying reasons that motivate it are common. When joining forces with another firm, an investment partner anticipates financial returns and/or a window on new technologies that correspond to future growth opportunities. ${ }^{1}$ As noted by Miller and Modigliani (1961), a significant

* Department of Civil, Architectural and Environmental Engineering, University of Padova, Via Marzolo 9, 35131, Padova, Italy. Email: dimitrios.zormpas@studenti.unipd.it. Special thanks to Michele Moretto and Luca Di Corato for valuable comments and suggestions.

${ }^{1}$ See e.g. Vrande and Vanhaverbeke (2013), Dushnitsky and Lenox (2005a) and Reuer and Tong (2007). 
part of many firms' market values consists of such future growth opportunities i.e. assets not yet in place. Myers (1977) argued that these assets are analogous to financial options, in the sense that one has the right but not the obligation to invest, and consequently stock option pricing methods should be used for their evaluation.

The real option approach acknowledges that investment opportunities are options on real assets and provides a way to apply option pricing methods to investment decision problems. It claims that the classic net present value rule is not always valid and argues that the option to postpone an investment decision characterized by uncertainty and irreversibility has to be taken into account. McDonald and Siegel (1986) gave an expression for the option value and showed that the optimal investment strategy is a trigger strategy in the sense that one should invest as soon as the project value is greater than a threshold, the value of which increases with uncertainty. ${ }^{2}$

In this paper we use the real option approach in order to describe the interaction among i) a firm who is contemplating entering an uncertain new market, ii) a firm who acts like an investment partner partly financing this project receiving a share of the final investment in return and iii) an upstream supplier with market power who is responsible for the provision of an input that is necessary for the potential investor to start producing and selling. Using a stochastic dynamic programming model, we examine how the involvement of the two alien agents affects the investment timing and how the observed timing discrepancies are reflected on the value of the option to invest. We show that both external funding and input outsourcing cause the postponement of the investment and we demonstrate how this erodes the project's option value. Comparing the two effects we find that external funding is preferred to input outsourcing, in the sense that the distortion with respect to the optimal, vertically integrated, case is more limited. We then focus on the three-agent case where the synchronous effect of outsourcing and external funding is discussed. Using a non-cooperative game-theoretic framework we show that, as expected, this represents the worst-case scenario since we have the investment timing reaching its maximum and, consequently, the value of the option to invest reaching its minimum. In the concluding part of the paper we approach the problem anew replacing the original non-cooperative game-theoretic setting with a Nash bargaining solution. Given a set of participation conditions, we show that the presence of the two alien agents is still affecting the investment timing. However, in this case the two effects are opposing each other. Outsourcing is still causing the postponement of the project but now external funding is favoring the (inefficient) acceleration of the investment. A comparison between the two shows that the first force is always prevailing, a result that highlights the importance of the nature of the sunk investment cost when investment partnerships are considered.

The remainder of the paper is organized as follows. In Section 2 we provide a brief overview of the related literature. In Section 3 we present in detail the model set-up demonstrating the connection with previous work and in Section 4 we discuss the three-agent case. In Section 5 we solve the problem under Nash bargaining and Section 6 concludes.

\section{Literature review}

This work relies on an established body of papers that integrate two research streams: the basic theory of irreversible investment under uncertainty as in Dixit and Pindyck (1994), and the classic presentation of vertical relationships as described e.g. by Tirole (1988). ${ }^{3}$ Real option analysis has been used to study joint ventures (Kogut, 1991; Li et al., 2008; Cvitanić et al., 2011; Banerjee et

\footnotetext{
${ }^{2}$ See e.g. Dixit and Pindyck (1994).

${ }^{3}$ Recent overviews of the related literature include Chevalier-Roignant et al., 2011; Azevedo and Paxson, 2014; Guimarães Dias and Teixeira, 2010; Huisman et al., 2004.
} 
al., 2014), R\&D and technology development (McGrath and Nerkar, 2004; McGrath, 1997; Folta, 1998), outsourcing (Alvarez and Stenbacka, 2007; Kogut and Kulatilaka, 1994; Moretto and Rossini, 2012; Di Corato et al., 2017; Triantis and Hodder, 1990; Teixeira, 2014), as well as venture capital investments (Lukas et al., 2016; Vrande and Vanhaverbeke, 2013; Dushnitsky and Lenox, 2005b) and acquisitions (Folta and Miller, 2002; Benson and Ziedonis, 2009; Lambrecht, 2004; Tong and Li, 2011).

The most closely related work we have identified is in corporate finance and supply chain management, most notably Lambrecht (2004), Banerjee et al. (2014) and Chen (2012), Lukas and Welling (2014).

Lambrecht (2004) analyzes a merger between two firms motivated by economies of scale using two different sequences of moves. According to the first (friendly merger), one of the two parties is choosing the optimal timing and then the terms of the merger are commonly decided, whereas, according to the second (hostile takeover), the two parties commit to the terms of the merger first and the timing is decided second. A comparison between the two suggests that, the way synergies are divided may influence the timing of the merger. Similarly, Banerjee et al. (2014) use a two-stage decision-making framework in which the parties determine the sharing rule as an outcome of Nash bargaining and one of them makes the timing decision related to the exercise of the jointly held option. Considering both cash transfers and ownership stakes, they show that when the exercise decision is made first, timing is always optimal ${ }^{4}$ whereas, when the sharing rule is determined first (hostile takeover case from Lambrecht, 2004) investment timing is socially inefficient unless a combination of stake in the project and a cash transfer is used. In this case, it generally matters which firm makes the timing decision and how the bargaining power is distributed.

In the supply chain management literature, Chen (2012) models a two-echelon supply chain consisting of one supplier and one retailer. The two-stage optimization problem evolves in the following way. In the first stage, the two agents negotiate over the optimal quantities whereas in the second stage, they coordinately determine the optimal timing of investing in the supply chain under uncertain demand. The results show that the volatility of demand shock has an ambiguous effect on the investment threshold with increasing impacts at lower level and decreasing impacts at higher level of uncertainty. Lastly, Lukas and Welling (2014) model the optimal timing of "climatefriendly" investments in a supply chain framework and enrich the contribution of Chen (2012) in the following ways. Firstly, they adopt a non-cooperative real option game setting according to which the optimal timing is decided, not jointly but by one of the participating firms and, secondly, they extend the two-echelon supply chain allowing for more than two participants showing that a supply chain becomes less efficient with every additional link as the timing distortion builds up.

In spite of the differences in their analyses, what all these papers have in common is the nature of the investment cost which is tacitly assumed to be exogenous. As Billette de Villemeur et al. (2014) point out, this assumption seems reasonable when the investment is performed largely inhouse, as may occur with R\&D, but this is not always the case. For instance, there are many other cases in which the completion of a firm's investment project depends on an upstream supplier who is responsible for the provision of a discrete input. In that case, the cost of the single firm's investment is endogenous since it is specified by the vertical relationship between the external supplier and the potential investor.

The novelty of our work lies on the fact that the investment cost is explicitly assumed to be endogenous. We begin by analyzing a non-cooperative game-theoretic setting according to which, the optimal timing is decided by the investment partner whereas the sharing rule is decided by the

\footnotetext{
${ }^{4}$ This is a generalization of the friendly merger case discussed in Lambrecht (2004) and in Morellec and Zhdanov (2005).
} 
project originator, given the price of an indispensable input provided by an external supplier with market power. We subsequently readdress this three-agent ${ }^{5}$ problem deriving the conditions under which it would be socially preferable to determine the sharing rule as an outcome of Nash bargaining and we find that the presence of the upstream firm makes a substantial difference when we consider the timing and the value of the option to invest in a given project, both in the cooperative (Nash bargaining solution) and in the non-cooperative case.

\section{The model}

\subsection{The basic set-up}

Firm $A$ is a risk neutral potential investor willing to enter a market with growing but uncertain demand. The profit flow that $A$ is cashing upon investment is $y_{t} \pi_{M}$ where $\pi_{M}>0$ is the instantaneous monopolistic profit per unit of $y_{t}$ and $y_{t}$ is a stochastic scale parameter that fluctuates according to the following geometric Brownian motion:

$$
\frac{d y_{t}}{y_{t}}=\alpha d t+\sigma d W_{t}, y_{0}=y
$$

where $\alpha>0$ is the drift, $\sigma>0$ is the instantaneous volatility and $d W_{t}$ is the standard increment of a Wiener process (standard Brownian motion) uncorrelated over time satisfying $E\left[d W_{t}\right]=0$ and $E\left[d W_{t}^{2}\right]=d t$

A discrete input is a prerequisite for $A$ to operate in the final market and this input is supplied by an upstream firm with market power that we call $C$. It is assumed that $C$ prices this input taking into consideration the structural parameters of the geometric Brownian motion presented above, but without ever observing $y_{t}{ }^{6}$

The completion of the project depends also on the cooperation of an investment partner $B$ who is willing to bear a share of the investment cost, asking for compensation in return. Contrary to the upstream firm, we assume that the investment partner is in a position to continuously and verifiably observe the fluctuations of the scale parameter over time. One can argue that this is a sensible assumption since $B$ will consider joining forces with $A$ only if s/he has enough information for the considered project. For instance, we can think of $B$ as a financial institution and $A$ as a customer asking for a business loan. The customer will need to present a thorough business plan in order to convince the financial institution about the promising character of the project. In this case, the potential investor is basically voluntarily sharing her/his information endowment with the investment partner. Alternatively, one can assume that $B$ might actually be in a position to observe the scale parameter over time without $A$ 's help. For instance, Mulherin and Boone (2000) as well as Vrande and Vanhaverbeke (2013) report evidence for significant industry clustering for merger and acquisition activities. These findings support the argumentation from Puranam et al. (2009) and Dushnitsky and Lenox (2005b) who suggest that firms seem to seek ventures similar to

\footnotetext{
${ }^{5}$ Note that our framework involves three agents of different type: the project originator, the investment partner and the input supplier. It is true that models with more than two agents have already been analyzed in the literature. For instance, Billette de Villemeur et al. (2014) present the case of a downstream duopoly with an upstream supplier and Banerjee et al. (2014) extend their two-party model to any number of investment partners. However, in both cases, as well as in the N-echelon supply chain presented by Lukas and Welling (2014), the types of agents are always two. The introduction of a third type is the key originality of our framework.

${ }^{6}$ As noted by Billette de Villemeur et al. (2014), if $C$ is in a position to continuously and verifiably observe the state of $y_{t}$, then $\mathrm{s}$ /he can expropriate the project by choosing a suitable input price. Here we assume that the upstream firm has no access to this kind of information.
} 
their own because this appears to facilitate the possible integration of technological resources in the future.

Before analyzing the three-agent case where the interaction among $A, B$ and $C$ is discussed, we briefly review the problem under (i) vertical integration and (ii) outsourcing or partial external funding (but not both).

\subsection{Vertical Integration}

The vertically integrated case will be our benchmark. In this simplified setting it is assumed that the potential investor produces the input in-house funding privately the completion of the project.

Following the real option literature, we keep in mind that there is some flexibility when we consider an investment opportunity under irreversibility and uncertainty. As firstly reported by McDonald and Siegel (1986), the ability to delay an irreversible investment expenditure is an important source of flexibility that profoundly affects the decision to invest. $A$ will only invest when the project's expected payoff exceeds the cost of the investment by the option value of waiting to invest. $^{7}$

Assuming that the initial market size is positive and sufficiently small so that a delay of the investment is preferable, the optimal investment time point $\tau^{V I}$ is derived through the solution of the following maximization problem:

$$
F_{A}^{V I}(y)=\max _{\tau^{V I}} E_{y}\left(\left(V_{\tau^{V I}}-I\right) e^{-r \tau^{V I}}\right)=\max _{y^{V I}}\left(\frac{y^{V I} \pi_{M}}{r-a}-I\right)\left(\frac{y}{y^{V I}}\right)^{\beta},
$$

where $V_{t}=E_{t}\left[\int_{t}^{\infty} y_{s} \pi_{M} e^{-r(s-t)} d s\right]=\frac{y_{t} \pi_{M}}{r-a}$ is the value of the project, $\beta \equiv \frac{1}{2}-\frac{a}{\sigma^{2}}+\sqrt{\left(\frac{a}{\sigma^{2}}-\frac{1}{2}\right)^{2}+\frac{2 r}{\sigma^{2}}}>$ 1 is the positive root of the characteristic equation $\frac{1}{2} \sigma^{2} \beta(\beta-1)+\alpha \beta-r=0, r(>\alpha)$ is the common to all firms discount rate, ${ }^{8} I>0$ is the sunk cost of producing the input in-house and $\tau^{V I}=\inf \left\{t \geq 0 \mid y_{t}=y^{V I}\right\}$ is the random first time point that $y_{t}$ hits the barrier $y^{V I}$ which is the market size that triggers the investment. The expressions for $F_{A}^{V I}(y)$ and $\beta$ are standard in the real option literature (see Dixit and Pindyck (1994), Chapters 5-6 and Dixit (1993), Section 2). From the first-order condition we have

$$
y^{V I}=\frac{\beta}{\beta-1} \frac{r-\alpha}{\pi_{M}} I .
$$

As expected, $y^{V I}$ is increasing in the sunk investment cost $I$ and the volatility $\sigma$ but is decreasing in the present value of the profit flow $\frac{\pi_{M}}{r-\alpha} \cdot{ }^{9}$ In words, a firm stands to gain more by holding, rather than exercising, an investment option with a high strike price ( $I$ in this case), a high underlying asset volatility ( $\sigma$ in this case) but small return $\left(\frac{\pi_{M}}{r-\alpha}\right.$ in this case). ${ }^{10}$

Combining Eq. (2) with Eq. (3) we obtain the value of the option to invest

$$
F_{A}^{V I}(y)=\frac{I}{\beta-1}\left(\frac{y}{y^{V I}}\right)^{\beta} .
$$

These results lead to the first proposition:

\footnotetext{
${ }^{7}$ O'Brien et al. (2003) present strong empirical evidence in favor of this argument. More precisely, they find that entrepreneurs account for the value of the option to delay entering a new market when contemplating such a decision.

${ }^{8}$ The inequality $r>\alpha$ guarantees convergence.

${ }^{9}$ Note that the effect of volatility on the investment threshold passes through $\beta$.

${ }^{10}$ Note that the classic net present value rule would dictate the lower investment threshold $y^{N P V}=\frac{r-\alpha}{\pi_{M}} I$. As one can see, even a risk neutral potential investor is sensitive to uncertainty when considering an irreversible investment the realization of which can be postponed for the future.
} 
Proposition 1 A single firm that produces the input in-house and self-finances the investment project, will enter the market as soon as the scale parameter reaches a threshold $y^{V I}=\frac{\beta}{\beta-1} \frac{r-\alpha}{\pi_{M}} I$. The value of the option to invest in this project is $F_{A}^{V I}(y)=\frac{I}{\beta-1}\left(\frac{y}{y^{V I}}\right)^{\beta}$.

\subsection{The input is outsourced and the investment is privately funded}

Suppose now that the input is produced by an upstream firm with market power that we call $C$. Adopting the framework presented by Billette de Villemeur et al. (2014), we assume that $A$ and $C$ engage in a leader-follower game at time zero. Moving backwards, $A$ (the follower) decides the optimal investment threshold taking into consideration the constant input price $p$. Then, $C$ (the leader) decides the optimal $p$ accounting for the production cost $I$, the structural parameters of the stochastic term $y_{t}$ and $A$ 's timing decision.

The optimal investment threshold is derived through the solution of the following maximization problem:

$$
F_{A}^{O S}(y)=\max _{y^{O S}(p)}\left(\frac{y^{O S}(p) \pi_{M}}{r-a}-p\right)\left(\frac{y}{y^{O S}(p)}\right)^{\beta}
$$

Solving we obtain

$$
y^{O S}(p)=\frac{\beta}{\beta-1} \frac{r-\alpha}{\pi_{M}} p .
$$

The decision problem of $C$, involves only the choice of $p$ that is derived as the solution of $F_{C}^{O S}(y)=$ $\max _{p^{O S}}\left(p^{O S}-I\right)\left(\frac{y}{y^{O S}\left(p^{O S}\right)}\right)^{\beta}$. Solving we have

$$
p^{O S}=\frac{\beta}{\beta-1} I
$$

Combining the optimal investment trigger from Eq. (6) with the optimal input price from Eq. (7), we obtain $y^{O S}\left(p^{O S}\right)=\frac{\beta}{\beta-1} \frac{r-\alpha}{\pi_{M}} \frac{\beta}{\beta-1} I$ which can be written as

$$
y^{O S}\left(p^{O S}\right)=\frac{\beta}{\beta-1} y^{V I}\left(>y^{V I}\right) .
$$

From Eq. (7) and Eq. (8) one can see that both $p^{O S}$ and $y^{O S}\left(p^{O S}\right)$ are decreasing in $\beta$ i.e. are increasing in volatility. This implies that $A$ will probably consider abandoning a very risky project or, in any case, will delay the investment as much as possible. ${ }^{11} C$ takes this into account setting a high $p^{O S}$ discounting this way for the delay between the time that $p^{O S}$ is chosen until the time it is cashed. Similarly, a project that involves very little risk will be undertaken relatively quickly by $A$ and this will also be reflected on a lower input price. Note however, that there is a minimum for $p^{O S}: \lim _{\sigma \rightarrow 0} p^{O S}=\frac{r}{r-a} I>I$. As one can see, despite the fact that the acceleration of the project is beneficial for $C, \mathrm{~s} /$ he is not willing to lower $p^{O S}$ below a minimum $\frac{r}{r-a} I$. This has to do with the fact that, for prices below $\frac{r}{r-a} I$, the completion of the project is indeed further hastened but what is sacrificed in terms of cash flow is not remunerated from the additional acceleration of the project.

Combining Eq. (5), (7) and (8) we obtain

$$
F_{A}^{O S}(y)=\left(\frac{\beta-1}{\beta}\right)^{\beta-1} F_{A}^{V I}(y) .
$$

\footnotetext{
${ }^{11} \lim _{\beta \rightarrow 1} y^{O S}\left(p^{O S}\right) \rightarrow \infty$
} 
Also, for $C$ we have

$$
F_{C}^{O S}(y)=\left(\frac{\beta-1}{\beta}\right)^{\beta} F_{A}^{V I}(y) .
$$

The following proposition summarizes these findings:

Proposition $2 A$ single firm that outsources the input and self-finances the investment project, will enter the market as soon as the scale parameter reaches a threshold $y^{O S}\left(p^{O S}\right)=\frac{\beta}{\beta-1} y^{V I}$. The value of the option to invest in this project is $F_{A}^{O S}(y)=\left(\frac{\beta-1}{\beta}\right)^{\beta-1} F_{A}^{V I}(y)$.

Comparing our findings from Propositions 1 and 2 we obtain the following:

Proposition 3 When an upstream supplier is responsible for the provision of the input, the investment is postponed $\left(y^{O S}>y^{V I}\right)$ and the potential investor's option to invest is less valuable $\left(F_{A}^{O S}(y)<F_{A}^{V I}(y)\right)$.

As one can see, the effect of the presence of $C$ is twofold. On one hand, it increases the investment threshold delaying the completion of the project and, on the other, it reduces the value of the option to invest for the potential investor $A$.

\subsection{The input is produced in-house and the investment is partly externally funded}

Going back to the case of vertical integration, we assume that the potential investor produces the input in-house. However, the realization of the project that $A$ has in mind depends now on the cooperation of an investment partner $B$.

Following Lukas and Welling (2014), $B$ is willing to undertake an exogenously given share $\xi \in(0,1)$ of the sunk investment cost, and this allows $A$ to fund only the rest of the project. In return, $A$ and $B$ negotiate over the compensation that the former needs to pay to the latter. More precisely, we assume that at time zero $A$ credibly commits to offer a fraction $\psi \in(0,1)$ of the project to $B$. Now $B$ has the option to accept this offer immediately disbursing the capital needed for the realization of the project or, alternatively, can delay this contribution for some future time. ${ }^{12}$ Similarly to the previous section, we have a leader-follower game with $A$ (the leader) deciding the compensation offer and $B$ (the follower) deciding the optimal timing taking the compensation offer $\psi$ into account. ${ }^{13}$

Starting with the problem of the follower we have $F_{B}^{V C}(y)=\max _{y^{V C}(\psi)}\left(\psi \frac{y^{V C}(\psi) \pi_{M}}{r-a}-\xi I\right)\left(\frac{y}{y^{V C}(\psi)}\right)^{\beta}$. Solving we obtain

$$
y^{V C}(\psi)=\frac{\xi}{\psi} y^{V I}
$$

\footnotetext{
${ }^{12}$ As one can see, the adopted framework is general enough to describe joint ventures and independent venture capital investments. However, it seems particularly suitable to describe corporate venture capital (CVC) investments. According to Roberts and Berry (1985), CVC investments consist of minority equity stakes in relatively new, not publicly traded companies and their purpose is to identify and value early-stage technology in start-ups. This description corresponds exactly to the behavior of our investment partner $B$ who agrees to receive a share $\psi$ of $A$ 's project instead of a standard cash flow.

${ }^{13}$ One can also consider the case where $B$ is the game-leader submitting the compensation offer and $A$ is the game-follower deciding the investment timing. In Section A.1 of Appendix A we show that such a change does not affect the nature of our main results.
} 
As expected, $y^{V C}(\psi)$ is increasing in $\xi$ and decreasing in $\psi$. This just means that the investment will be postponed as the cost share for the investment partner is increasing, a result that can be neutralized if the potential investor is willing to improve the submitted compensation offer. As one can see, $A$ faces a dilemma since a low compensation offer implies access to a larger cash flow later in the future whereas a high compensation offer shortens the waiting period but gives access to a smaller cash flow in return.

The potential investor takes into consideration the reaction of the investment partner and decides the compensation offer that is derived as the solution of the following maximization problem:

$$
F_{A}^{V C}(y)=\max _{\psi^{V C}}\left(\left(1-\psi^{V C}\right) \frac{y^{V C}\left(\psi^{V C}\right) \pi_{M}}{r-a}-(1-\xi) I\right)\left(\frac{y}{y^{V C}\left(\psi^{V C}\right)}\right)^{\beta}
$$

which yields

$$
\psi^{V C}=\frac{\xi(\beta-1)}{\beta-1+\xi} .
$$

Combining Eq. (11) and Eq. (13) we obtain

$$
y^{V C}\left(\psi^{V C}\right)=\frac{\beta-1+\xi}{\beta-1} y^{V I}\left(>y^{V I}\right) .
$$

Studying the optimal compensation offer, we can see that $\psi^{V C}$ is increasing both in $\beta$ and in $\xi$. Focusing on the effect of $\xi$, it is interesting to see that the maximum optimal offer is always below $100 \%$. This has to do with the fact that a more generous compensation offer will indeed hasten the completion of the project but will only make the potential investor worse-off since what is sacrificed in terms of compensation is not remunerated from the acceleration of the investment.

One can also observe that $y^{V C}\left(\psi^{V C}\right)$ is increasing in $\xi$ and decreasing in $\beta$. Actually, the "distance" between $y^{V C}\left(\psi^{V C}\right)$ and $y^{V I}$ increases in $\xi$ despite the fact that, at the same time, $\psi^{V C}$ is also increasing in $\xi$. This happens because $y^{V C}\left(\psi^{V C}\right)$ is a linear, whereas $\psi^{V C}$ is a concave function of $\xi$. In words, despite the fact that the compensation offer is becoming more generous as the share of the cost covered by $B$ increases, in real terms the offer worsens and this is reflected on a higher investment threshold.

Lastly, combining Eq. (12), (13) and (14) we obtain

$$
F_{A}^{V C}(y)=\left(\frac{\beta-1}{\beta-1+\xi}\right)^{\beta-1} F_{A}^{V I}(y)
$$

Also, for $B$ we have

$$
F_{B}^{V C}(y)=\xi\left(\frac{\beta-1}{\beta-1+\xi}\right)^{\beta} F_{A}^{V I}(y)
$$

The following proposition summarizes these results.

Proposition 4 In the case where the single firm produces the input in-house but the completion of the project depends on external funding, the investment occurs when the scale parameter reaches a threshold $y^{V C}\left(\psi^{V C}\right)=\frac{\beta-1+\xi}{\beta-1} y^{V I}$. The value of the option to invest in this project is $F_{A}^{V C}(y)=$ $\left(\frac{\beta-1}{\beta-1+\xi}\right)^{\beta-1} F_{A}^{V I}(y)$.

Comparing our findings with the values that we derived in the vertically integrated case we have: 
Proposition 5 In the case where the single firm produces the input in-house but the completion of the project depends on external funding, the investment is delayed $\left(y^{V C}>y^{V I}\right)$ and the potential investor's option to invest is less valuable $\left(F_{A}^{V C}(y)<F_{A}^{V I}(y)\right)$.

As in Section 3.3, the effect of the presence of the additional agent is twofold since, on one hand, it increases the investment threshold delaying the completion of the project and, on the other, it reduces the value of the option to invest for the potential investor.

\section{The three-agent case}

In this section we combine the analyses presented above and we show how the synchronous presence of $B$ and $C$ affects the performance and the actions of $A$ as well as the investment threshold. The three-agent game evolves in the following way:

1. $C$ is the game-leader and decides the input price that maximizes her/his value of the option to invest.

2. Given the input price and the cost share $\xi, A$ submits the compensation offer $\psi$ to $B$ and, finally,

3. $B$ evaluates this compensation offer and decides when to accept it, disbursing the amount that is required for the realization of $A$ 's project.

Keeping in mind that $A$ and $B$ continuously and verifiably observe the magnitude of $y_{t}$ whereas $C$ only knows the structural parameters of the related stochastic process, we move backwards and we first study the behavior of the investment partner $B$. The optimal investment threshold is derived through the solution of: $F_{B 3}(y)=\max _{y_{3}(\psi, p)}\left(\psi \frac{y_{3}(\psi, p) \pi_{M}}{r-a}-\xi p\right)\left(\frac{y}{y_{3}(\psi, p)}\right)^{\beta}$. Solving, we obtain

$$
y_{3}(\psi, p)=\frac{\beta}{\beta-1} \frac{r-\alpha}{\pi_{M}} p \frac{\xi}{\psi}
$$

The potential investor $A$ will take into consideration the decision of the investment partner and will choose the compensation offer taking the price of the input as given. The optimal $\psi$ is derived as the solution of $F_{A 3}(y)=\max _{\psi_{3}}\left(\left(1-\psi_{3}\right) \frac{y_{3}\left(\psi_{3}, p\right) \pi_{M}}{r-a}-(1-\xi) p\right)\left(\frac{y}{y_{3}\left(\psi_{3}, p\right)}\right)^{\beta}$. From the first-order condition we have

$$
\psi_{3}=\frac{\xi(\beta-1)}{\beta-1+\xi} .
$$

As one can check, this is exactly the compensation offer that we derived in Eq. (13) where $C$ was absent. Obviously, the presence/absence of $C$ does not affect the magnitude of the optimal compensation offer that $A$ submits to $B$ since the exogenously given cost share $\xi$ has to do with the generic investment cost no matter if this is $I$ or $p$.

We conclude with the game-leader $C$. The input supplier observes the behavior both of $A$ and of $B$ and optimally decides the price of the input solving $F_{C 3}(y)=\max _{p_{3}}\left(p_{3}-I\right)\left(\frac{y}{y_{3}\left(\psi_{3}, p_{3}\right)}\right)^{\beta}$ which yields:

$$
p_{3}=\frac{\beta}{\beta-1} I
$$

Comparing Eq. (19) with Eq. (7) we see that $p_{3}=p^{O S}$ which means that the presence/absence of $B$ does not affect the optimal price of the input that is decided by $C$. This is not a surprise since, $C$ is indifferent to the means that $A$ uses to fund the project. 
Combining Eq. (17), (18) and (19) we derive the investment threshold which, in this case, is

$$
y_{3}\left(\psi_{3}, p_{3}\right)=\frac{\beta}{\beta-1} \frac{\beta-1+\xi}{\beta-1} y^{V I}\left(>y^{V I}\right) .
$$

Note that $y_{3}\left(\psi_{3}, p_{3}\right)$ is decreasing in $\beta$ and increasing in $\xi$. Similarly to $y^{V C}\left(\psi^{V C}\right)$, the "distance" between $y_{3}\left(\psi_{3}, p_{3}\right)$ and $y^{V I}$ increases in $\xi$ in spite of the simultaneous improvement of the corresponding compensation offer. The reasoning is the same: the investment trigger is a linear whereas the compensation offer is a concave function of the cost share $\xi$. As we have seen in the previous section, despite the fact that the compensation offer is becoming more generous as the cost share $\xi$ increases, in real terms the offer worsens and actually, in the three-agent case, this effect is even more dramatic since the compensation offer worsens "faster" as the (positive) slope of $y_{3}$ with respect to $\xi$ is larger than the (positive) slope of $y^{V C}$ with respect to $\xi$ exactly because of the presence of $C$.

We conclude this section returning to the option values for the three agents. Keeping in mind the values for $y_{3}\left(\psi_{3}, p_{3}\right), p_{3}$ and $\psi_{3}$ we obtain

$$
\begin{aligned}
& F_{A 3}(y)=\left(\frac{\beta-1}{\beta}\right)^{\beta-1}\left(\frac{\beta-1}{\beta-1+\xi}\right)^{\beta-1} F_{A}^{V I}(y), \\
& F_{B 3}(y)=\xi\left(\frac{\beta-1}{\beta}\right)^{\beta-1}\left(\frac{\beta-1}{\beta-1+\xi}\right)^{\beta} F_{A}^{V I}(y), \\
& F_{C 3}(y)=\left(\frac{\beta-1}{\beta}\right)^{\beta}\left(\frac{\beta-1}{\beta-1+\xi}\right)^{\beta} F_{A}^{V I}(y) .
\end{aligned}
$$

The following proposition summarizes these results.

Proposition 6 In the three-agent case where the single firm outsources the production of the input and the completion of the project depends on external funding, the investment occurs when the scale parameter reaches a threshold $y_{3}\left(\psi_{3}, p_{3}\right)=\frac{\beta}{\beta-1} \frac{\beta-1+\xi}{\beta-1} y^{V I}$. The value of the option to invest in this project for the potential investor is $F_{A 3}(y)=\left(\frac{\beta-1}{\beta}\right)^{\beta-1}\left(\frac{\beta-1}{\beta-1+\xi}\right)^{\beta-1} F_{A}^{V I}(y)$.

Comparing our findings with the values that we derived in the vertically integrated case we have:

Proposition 7 In the three agent case the investment takes place inefficiently late $\left(y_{3}\left(\psi_{3}, p_{3}\right)>y^{V I}\right)$ and this is also reflected on the potential investor's option value $\left(F_{A 3}(y)<F_{A}^{V I}(y)\right)$.

\subsection{Discussion}

In the previous sections we focused on the effect that the presence of additional agents has on the investment threshold and the potential investor's option value both for the two-agent and the three-agent case. Keeping all this in mind we can examine this effect in the level of the industry as a whole.

The input is outsourced and the investment is privately funded: We have already computed the value of the option to invest for the potential investor $A$ and the input supplier $C$. Now adding up Eq. (9) and Eq. (10) we obtain

$$
F^{O S}(y) \equiv F_{A}^{O S}(y)+F_{C}^{O S}(y)=\frac{(\beta-1)^{\beta-1}}{\beta^{\beta}}(2 \beta-1) F_{A}^{V I}(y)
$$


As one can see, the presence of the firm $C$ affects negatively the value of the option to invest for the whole industry since $F^{O S}(y)<F_{A}^{V I}(y)$.

The input is produced in-house and the investment is partly externally funded: Summing Eq. (15) and Eq. (16) we obtain

$$
F^{V C}(y) \equiv F_{A}^{V C}(y)+F_{B}^{V C}(y)=\frac{\beta-1+\xi \beta}{\beta-1}\left(\frac{\beta-1}{\beta-1+\xi}\right)^{\beta} F_{A}^{V I}(y) .
$$

The presence of firm $B$ affects negatively the value of the option to invest for the whole industry since $F^{V C}(y)<F_{A}^{V I}(y)$.

The three-agent case: Summing up the values of the option to invest for $A, B$ and $C$ from Eq. (21) we obtain

$$
\begin{aligned}
F_{3}(y) & \equiv F_{A 3}(y)+F_{B 3}(y)+F_{C 3}(y) \\
& =\left(\frac{\beta-1}{\beta}\right)^{\beta-1}\left(\frac{\beta-1}{\beta-1+\xi}\right)^{\beta}\left(\frac{\beta-1+\xi}{\beta-1}+\xi+\frac{\beta-1}{\beta}\right) F_{A}^{V I}(y) .
\end{aligned}
$$

A comparison of the results derived in this and the previous sections is given in the following proposition:

Proposition 8 A comparison among the investment triggers and the option values presented above gives the following rankings:

1) $y_{3}\left(\psi_{3}, p_{3}\right)>y^{O S}\left(p^{O S}\right)>y^{V C}\left(\psi^{V C}\right)>y^{V I}$,

2) $F_{3}(y)<F^{O S}(y)<F^{V C}(y)<F_{A}^{V I}(y)$,

3) $F_{A 3}(y)<F_{A}^{O S}(y)<F_{A}^{V C}(y)<F_{A}^{V I}(y)$,

4) $F_{B 3}(y)<F_{B}^{V C}(y)$ and

5) $F_{C 3}(y)<F_{C}^{O S}(y)$.

A number of interesting results can be derived by these comparisons. First of all, we see that as the number of agents involved in an investment project increases, the completion of this project is postponed to the detriment of the investment's option value both in the firm and in the industry level. As expected, the vertically integrated case represents the most favorable whereas the threeagent case represents the least favorable scenario. Another interesting observation has to do with the comparison between the effect of the presence of the upstream firm $C$ and the effect of the presence of the investment partner $B$. As we can see, external funding is preferred to outsourcing in terms of timing and, consequently, in terms of option value. ${ }^{14}$

\subsection{Numerical Examples}

We conclude this section using some numerical examples that will help us illustrate the effect of outsourcing and external funding on the investment timing and the value of the option to enter the new market.

\footnotetext{
${ }^{14}$ In Section A.2 of Appendix A we show that the ranking of the investment thresholds and, consequently, the ranking of the aggregate option values, is the same even when $A$ and $B$ swap places i.e. if $A$ becomes the timedeciding agent (game-follower) and $B$ becomes the one that chooses the compensation share (game-leader). However, we also show that this is not the case for the ranking of $A$ 's option values which is actually sensitive to such a change.
} 
We assume that the parameters vary as follows: We let the drift, $\alpha$, and the volatility, $\sigma$, take values $\{0.025,0.035\}$ and $\{0.2,0.25,0.3,0.35,0.4\}$ respectively. A high magnitude of the drift captures a high expected increase in the size of the new market whereas different levels of volatility are used to demonstrate the impact of uncertainty on the investment thresholds and option values. The sunk investment cost $I$ takes values $\{24,48\}$ whereas both the initial level of the stochastic parameter $y_{0}$ and the instantaneous monopolistic profit per unit of $y_{t}, \pi_{M}$, are set equal to unity $\left(y_{0}=\pi_{M}=1\right)$. We allow for three different levels of exogenous cost share: $\xi_{1}=0.1, \xi_{2}=0.5$ and $\xi_{3}=0.9$ in order to demonstrate how the participation of an investment partner affects the timing and the performance of the investment project. The interest rate $r$ is initially set equal to $5 \%$ but we also check the effect of an increase to $6 \%$ which corresponds to a higher opportunity cost of capital.

In Table 1, we use $\alpha=0.025, \sigma=0.2, r=0.05, y_{0}=1, \xi=0.5$ and $I=24$ and we obtain the values that serve as our standard of comparison. As one can check, Table 1 demonstrates how the investment threshold and the value of the option to invest in a given project are affected by the presence of an investment partner and/or the presence of an upstream firm. In accordance to Proposition 8, we see that the presence of any alien agent causes the procrastination of the investment. The investment threshold is, roughly, doubled when an investment partner is involved, and tripled when a necessary input is outsourced. However, the combined effect is more dramatic since, under both outsourcing and external funding, the investment threshold is more than six times higher than the investment threshold under vertical integration.

As for the project value itself, we see that the changes in the investment threshold are also reflected on the value of the option to invest. The project depreciates whenever its completion depends on the contribution of one of the other two parties, with outsourcing being less preferable than an investment partnership but more preferable than the three-agent case. Interestingly, under external funding the investor's share of the project is equal to $71 \%$ of her/his share under vertical integration whereas, when outsourcing is explicitly taken into consideration the percentage drops to $42 \%$. Unsurprisingly, a similar effect is observed when one studies the value of the option to invest in the level of the industry. Arguably, these results underline the difference that the nature of the investment cost (endogenous or exogenous) can make when an investment project characterized by uncertainty and irreversibility is considered.

\section{TABLE 1}

Standard of Comparison

\begin{tabular}{|c|c|c|c|c|}
\hline & Vertical Integration & External Funding & Outsourcing & Three-Agent Case \\
\hline$y^{*}$ & 1.90 & 3.96 & 6.02 & 12.55 \\
\hline$F_{A}$ & 20.35 & 14.50 & 11.96 & 8.52 \\
\hline$F_{B}$ & - & 3.48 & - & 2.04 \\
\hline$F_{C}$ & - & - & 3.77 & 1.29 \\
\hline$F$ & 20.35 & 17.98 & 15.73 & 11.85 \\
\hline
\end{tabular}

In Table 2 we examine the effect that a change in the cost $I$ has on the investment thresholds and the option values presented above. For $I=48$, i.e. for an investment cost two times higher than our standard of comparison, all the investment thresholds are doubled in magnitude and all the option values drop to, about, $73 \%$ of their corresponding values given in Table 1. Starting with the investment thresholds, it is evident that a potential investor will have less rush to invest in a, other things equal, more expensive project. As for the depreciation of the option values, one can argue that two opposing forces drive this result. On one hand, the value of the option to delay a costly and irreversible investment is valuable, and this value is expected to increase in $I$ since, the 
more expensive the investment, the more valuable the option to postpone it. On the other hand however, the higher investment threshold implies a further delay of the investment which eventually distances the anticipated cash flow further in the future. As one can see, the second force prevails.

\section{TABLE 2}

The Effect of a Change in the Investment Cost on Timing and Option Value

\begin{tabular}{|c|c|c|c|c|}
\hline$I=48$ & & & & \\
\hline & Vert. Integ. & Ext. Funding & Outsourcing & Three-Agent Case \\
\hline$y^{*} / y_{T 1}^{*}$ & 2 & 2 & 2 & 2 \\
\hline$F_{A} / F_{A}^{T 1}$ & 0.73 & 0.73 & 0.73 & 0.73 \\
\hline$F_{B} / F_{B}^{T 1}$ & - & 0.73 & - & 0.73 \\
\hline$F_{C} / F_{C}^{T 1}$ & - & - & 0.73 & 0.73 \\
\hline$F / F^{T 1}$ & 0.73 & 0.73 & 0.73 & 0.73 \\
\hline
\end{tabular}

In Table 3, we study the effect that changes in the drift, $\alpha$, may have. A comparison of the option values of Table 3 with the ones that we derived in Table 1 shows that an increase in the expected growth rate from $\alpha=0.025$ to $\alpha=0.035$, is beneficial both in the firm and in the industry level. However, the effect of such a change in the investment triggers is not as obvious. Actually, we observe that a higher $\alpha$ is, ceteris paribus, encouraging the acceleration of a project under vertical integration but is causing the further postponement of projects the completion of which is conditional on the participation of a second or a third party. Especially, in the three-agent case, we see that a $\Delta \alpha=0.01$ is enough to (more than) double the related investment threshold. The intuition behind this result has to do exactly with the absence or the presence of the alien firms. When the potential investor acts unilaterally, a positive change in $\alpha$ signals the shortening of the expected waiting period until the right time for the investment to take place has come. This, of course, is reflected on a lower investment threshold. ${ }^{15}$ Nevertheless, under the presence of an upstream supplier and/or an investment partner, the situation is quite different. The upstream firm updates the price of the input asking a higher price whereas the compensation offer that the investment partner receives is now readjusted for the higher $\alpha$. Eventually, the time-deciding agent accounts for these changes choosing a higher, instead of lower, investment threshold.

\section{TABLE 3}

The Effect of a Change in the Drift on Timing and Option Value

\begin{tabular}{|c|c|c|c|c|}
\hline$\alpha=0.035$ & & & & \\
\hline & Vert. Integ. & Ext. Funding & Outsourcing & Three-Agent Case \\
\hline$y^{*}$ & 1.80 & 5.40 & 9 & 27 \\
\hline$F_{A}$ & 46.04 & 34.98 & 30.79 & 23.39 \\
\hline$F_{B}$ & - & 5.83 & - & 3.89 \\
\hline$F_{C}$ & - & - & 6.15 & 1.55 \\
\hline$F$ & 46.04 & 40.81 & 36.94 & 28.83 \\
\hline
\end{tabular}

As for the volatility $\sigma$, in Figure 1 and Figure 2 we see how an increase in $\sigma$ from $\sigma=0.2$ to $\sigma=0.4$ affects the timing and the value of the option to invest in the project under consideration.

\footnotetext{
${ }^{15}$ As we can see from Eq. (3), this is actually the effect of two opposing forces. On one hand, a higher $a$ implies a higher present value for the profit flow $\left(\frac{\pi_{M}}{r-\alpha}\right)$ which, in return, favors the acceleration of the investment. On the other hand however, an increased drift implies a lower $\frac{\beta}{\beta-1}$ which is the factor that corrects the investment threshold for uncertainty and irreversibility. Apparently, under vertical integration, the first force prevails.
} 
As expected, all the investment thresholds are increasing in $\sigma$. As is standard in the real option literature, the presence of uncertainty and irreversibility calls for a more careful investment policy. Hence, investment projects should occur at higher return levels, a decision which in turn requires waiting longer before investing. Note however that higher investment thresholds do not cause the depreciation of the value of the corresponding project. On the contrary, a higher volatility is, as anticipated, increasing the value of the option to delay the completion of an investment project characterized by uncertainty and irreversibility.

What is interesting to see here is that the investment threshold in the three-agent case is significantly more sensitive to an increase in volatility with respect to the other three investment scenarios that we study, a result which is also reflected on a proportionately higher option value. As before, the intuition here is that the investment threshold under both outsourcing and external funding is affected both by the decisions of the project originator and by the actions of the investment partner and the upstream firm which are also affected by changes in $\sigma$. For instance, we know that the upstream firm anticipates that an increase in $\sigma$ will further delay the completion of the investment project. This foresight calls for the decision of a higher input price that will act as compensation against this (further) delayed cash flow. Similarly, the project originator also anticipates a (further) postponement in the completion of the project and, using analogous reasoning, decides to decrease the submitted compensation offer. Finally, the time-deciding investment partner observes the increase in $\sigma$ as well as the updated input price and compensation offer and chooses an appropriately high investment threshold that accounts for all this.

\section{FIGURE 1}

The Effect of a Change in Volatility on the Investment Threshold

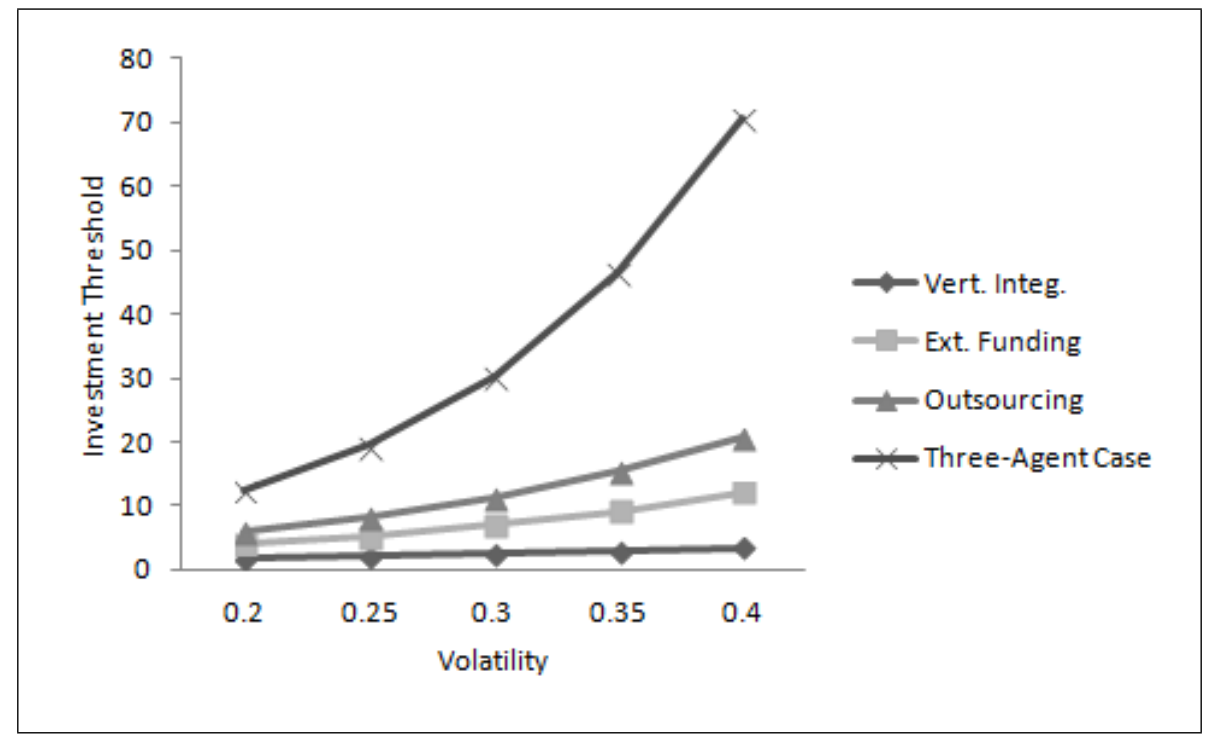




\section{FIGURE 2}

The Effect of a Change in Volatility on the Option Value

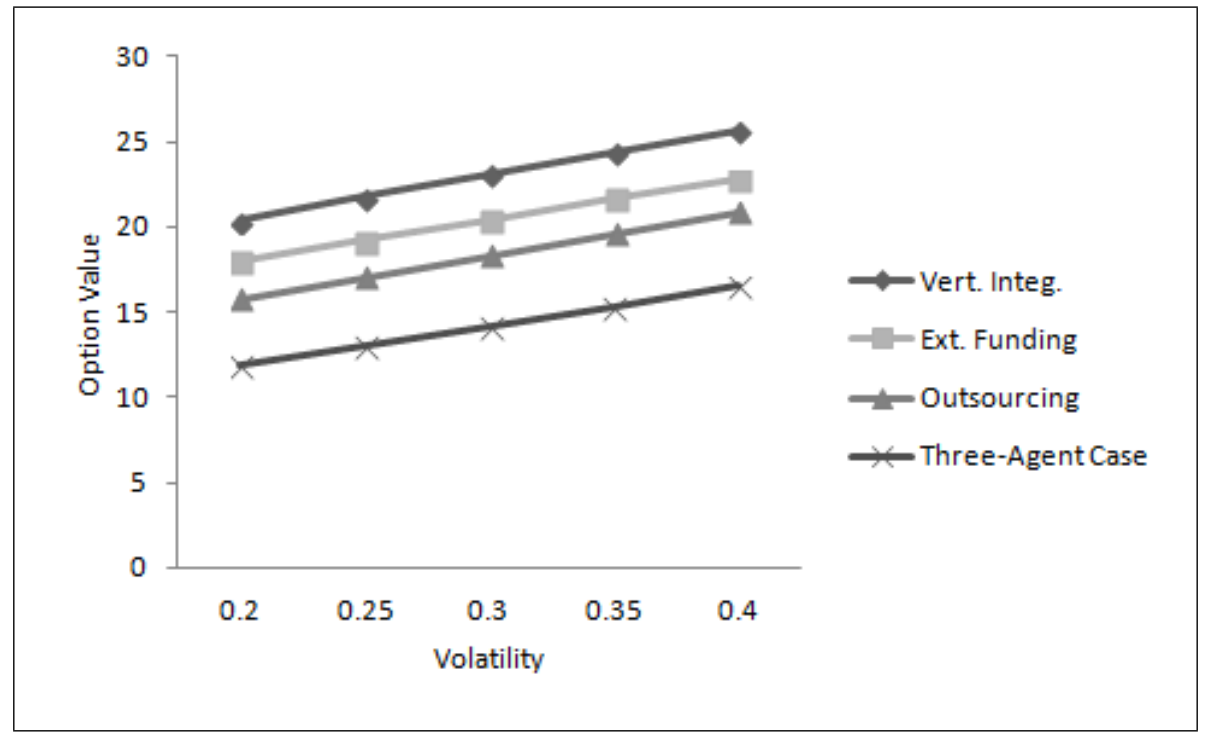

TABLE 4

The Effect of a Change in the Exogenous Cost Share on Timing and Option Value

\begin{tabular}{|c|c|c|c|c|}
\hline & \multicolumn{2}{|c|}{$\xi=0$} & \multicolumn{2}{c|}{$\xi=0.1$} \\
\hline & Ext. Funding & Three-Agent Case & Ext. Funding & Three-Agent Case \\
\hline$y^{*}$ & 1.90 & 6.02 & 2.31 & 7.33 \\
\hline$F_{A}$ & 20.35 & 11.96 & 18.60 & 10.92 \\
\hline$F_{B}$ & 0 & 0 & 1.53 & 0.90 \\
\hline$F_{C}$ & - & 3.77 & - & 2.83 \\
\hline$F$ & 20.35 & 15.73 & 20.13 & 14.65 \\
\hline \hline & \multicolumn{3}{|c|}{$\xi=0.5$} & \multicolumn{2}{c|}{$\xi=0.9$} \\
\hline & Ext. Funding & Three-Agent Case & Ext. Funding & Three-Agent Case \\
\hline$y^{*}$ & 3.97 & 12.56 & 5.61 & 17.79 \\
\hline$F_{A}$ & 14.50 & 8.52 & 12.35 & 7.26 \\
\hline$F_{B}$ & 3.48 & 2.04 & 3.77 & 2.21 \\
\hline$F_{C}$ & - & 1.29 & - & 0.78 \\
\hline$F$ & 17.98 & 11.85 & 16.12 & 10.25 \\
\hline
\end{tabular}

In Table 4 we focus on the impact that a change in the exogenous investment cost share $\xi$ may have. The benchmark value that we choose is $\xi=0.5$ which implies a perfectly balanced investment scheme with both partners undertaking equal portions of the sunk cost. We subsequently allow both for high $(\xi=0.9)$ and for low $(\xi=0.1)$ investment cost shares and we also present, for comparison's sake, the case where there is no partnership $(\xi=0)$. Starting with the investment thresholds, we note that a higher involvement of an investment partner always implies the postponement of the project. Of course, keeping in mind the analysis of Section 3.4 and Section 4.1, this is hardly a surprise. As we have already seen there, a higher cost share $\xi$ implies a higher nominal, but lower real, compensation offer from the project originator to the investment partner. Eventually, this is reflected on a higher investment threshold and the further procrastination of the investment. 
The effect of a change in $\xi$ on the option values of the three parties is nothing but an extension of the effect that we observe in the investment triggers. A higher $\xi$ causes the depreciation of the value of the option to invest for every party apart from the investment partner who is favored by such a change. This adverse effect is also clearly reflected on the option value of the industry as a whole.

Lastly, in Table 5 we study the effect of a change in the interest rate $r$. We start with the vertically integrated case. As far as the investment threshold is concerned, two opposing forces are acting. On one hand, an increase in $r$ makes the potential investor more impatient since, with a higher interest rate, the present becomes relatively more important than the future which implies the selection of a lower investment threshold. At the same time however, the increase in $r$ implies a decrease in the present value of the profit flow that the project is meant to generate once it takes place. This limits the interest of the potential investor to invest right now in a project which does not cover the high opportunity cost of capital. As we see in Table 5, the second force prevails causing the postponement of the investment.

In the case where we deal with an investment partnership, an increase in the interest rate from 0.05 to 0.06 causes a similar effect but of smaller magnitude. The analysis of the previous paragraph holds here as well. However, we need to take into account the fact that the investment trigger is now also affected by the change in the compensation offer that is submitted to the time-deciding agent. The project originator, being impatient her/himself, is willing to make a more generous compensation offer in an attempt to shorten the waiting period till the completion of the project. As one can see, this makes a difference almost neutralizing the increase in $r$.

The most interesting cases involve the participation of the upstream firm. Despite the fact that the argumentation from above still applies, the presence of an impatient upstream firm causes, as one can see in Table 5, the acceleration of the investment. In order to understand the intuition behind this result, one should keep in mind that the effect of a change in $r$ is different for the upstream firm than it is for the two investment partners. It is true that all the involved firms discount the value of the option to invest with a common discount factor. ${ }^{16}$ However, the way that each agent evaluates the net present value of the project at the delivery date is different. For the two investment partners, the completion of the investment project signals the commencing of a profit flow that needs to be appropriately discounted. Of course, a change in $r$ affects the chosen discounting factor. On the contrary, when the delivery date is reached, the upstream firm receives a lump sum which corresponds to the price of the input that s/he supplied and which is not affected by changes in $r$. As a consequence, even a small increase in the discount rate is enough to make the upstream supplier sufficiently impatient and willing to ask a lower input price as soon as this will lead to the acceleration of the investment. Indeed, in Table 5 we see that, when an upstream firm is present, an increased discount rate encourages the acceleration of the completion of the project, a result which is most prevalent in the three-agent case where the impatience of the two alien agents concurs.

As for the value of the option to invest, we see that even a slightly increased interest rate can considerably reduce the project's option value. As we have already stressed above, an increased interest rate implies that the present becomes financially more important than the future. Hence,

\footnotetext{
${ }^{16}$ Recall that in Section 3.3 we have $\left(\frac{y}{y^{O S}\left(p^{O S}\right)}\right)^{\beta}$ both for $A$ and for $C$, in Section 3.4 we have $\left(\frac{y}{y^{V C}\left(\psi^{V C}\right)}\right)^{\beta}$ both for $A$ and for $B$ and in Section 4.1 we have $\left(\frac{y}{y_{3}\left(\psi_{3}, p_{3}\right)}\right)^{\beta}$ for all three agents.
} 
the option to delay an investment project for some future time point naturally becomes less valuable.

TABLE 5

The Effect of a Change in the Interest Rate on Timing and Option Value

\begin{tabular}{|c|c|c|c|c|}
\hline$r=0.06$ & & & & \\
\hline & Vert. Integ. & Ext. Funding & Outsourcing & Three-Agent Case \\
\hline$y^{*} / y_{T 1}^{*}$ & 1.16 & 1.02 & 0.97 & 0.84 \\
\hline$F_{A} / F_{A}^{T 1}$ & 0.54 & 0.52 & 0.50 & 0.49 \\
\hline$F_{B} / F_{B}^{T 1}$ & - & 0.60 & - & 0.56 \\
\hline$F_{C} / F_{C}^{T 1}$ & - & - & 0.60 & 0.68 \\
\hline$F / F^{T 1}$ & 0.54 & 0.54 & 0.53 & 0.52 \\
\hline
\end{tabular}

\section{The compensation as the product of Nash bargaining}

In Section 3.4 and in Section 4 we used a non-cooperative setting in order to describe the interaction between the potential investor $A$ and the investment partner $B$. However, as noticed by the extant literature, co-development partnerships are an increasingly utilized way of improving profitability, competitiveness and innovation effectiveness. ${ }^{17}$ In the following, we will attempt to re-approach the potential investor's business plan using a cooperative framework. More precisely, we assume that the compensation offer will now be replaced by a Nash bargaining solution that will explicitly reflect the bargaining power of the involved agents. We begin with the two-agent case and we subsequently allow for outsourcing.

\subsection{The input is produced in-house and the investment is partly externally funded}

Similarly to the presentation of Section 3.4, we assume that $A$ can produce the input in-house and that the completion of the project is conditional on the participation of a firm $B$ who acts like an investment partner. As before, $B$ is willing to undertake a share $\xi$ of the sunk investment cost given that $\mathrm{s} /$ he will receive compensation in return. What is new here with respect to the analysis of Section 3.4 is that, by assumption, $A$ agrees with $B$ on the compensation share and then decides the optimal investment threshold. Note that, contrary to the initial analysis, we now assume that $A$, not $B$, is the time deciding agent. Apparently, $A$ sacrifices her/his exclusivity on the decision of $\psi$ in order to become the time-deciding agent and similarly $B$ sacrifices her/his position as the time-deciding agent in order to have a say in the decision of $\psi \cdot{ }^{18}$ In the following we see under what conditions this cooperative framework can replace the non-cooperative one.

Starting with the maximization problem of the time-deciding agent we have:

$$
F_{A N}(y)=\max _{y_{N}(\psi)}\left((1-\psi) \frac{y_{N}(\psi) \pi_{M}}{r-a}-(1-\xi) I\right)\left(\frac{y}{y_{N}(\psi)}\right)^{\beta} .
$$

Solving we obtain

$$
y_{N}(\psi)=\frac{1-\xi}{1-\psi} y^{V I}
$$

\footnotetext{
${ }^{17}$ See Zhou and Yang (2008), Biehl et al. (2006) and Cvitanić et al. (2011) respectively.

${ }^{18}$ In Appendix A we show how the analysis presented in Section 3.4 and Section 4 changes if $A$ and $B$ swap places. In Appendix B we do the same for the analysis presented in Section 5 where the initial non-cooperative game-theoretic framework is replaced by a Nash bargaining solution and we show that such a change does not affect the nature of our main results.
} 
Moving one step back, the two parties bargain anticipating that $A$ will invest as soon as $y_{t}$ reaches the trigger $y_{N}(\psi)$. Given this, the new optimal compensation share is derived as the solution of

$$
\max _{\psi_{N}} \frac{\left(\psi_{N} \frac{y_{N}\left(\psi_{N}\right) \pi_{M}}{r-a}-\xi I\right)^{\eta_{B}}}{\left(\left(1-\psi_{N}\right) \frac{y_{N}\left(\psi_{N}\right) \pi_{M}}{r-a}-(1-\xi) I\right)^{\eta_{B}-1}}\left(\frac{y}{y_{N}\left(\psi_{N}\right)}\right)^{\beta},
$$

where $\eta_{B}$ represents $B$ 's bargaining power. ${ }^{19}$ Solving we obtain

$$
\psi_{N}=\frac{\xi(\beta-1)+\eta_{B}(1-\xi)}{\beta-\xi} .
$$

Combining Eq. (26) and Eq. (28) we have

$$
y_{N}\left(\psi_{N}\right)=\frac{\beta-\xi}{\beta-\eta_{B}} y^{V I}
$$

One can check that the compensation $\psi_{N}$ increases linearly in $\eta_{B} \cdot{ }^{20}$ It is also true that, contrary to the compensation offers that we have encountered in the previous sections, $\psi_{N}$ is not always increasing in $\beta$, hence decreasing in volatility. More precisely, here we have $\frac{\partial \psi_{N}}{\partial \beta} \gtrless 0$ for $\xi \gtrless \eta_{B}$. In words, the compensation offer is decreasing in volatility only when the bargaining power of $B$ is sufficiently low. Another interesting point is that when $B$ bears almost the whole investment cost $(\xi \rightarrow 1)$, we obtain $\psi_{N} \simeq 1$. Contrary to the compensation offer $\psi^{V C}$ that cannot be larger than $\frac{\beta-1}{\beta}, \psi_{N}$ can reach values as high as $100 \%$, irrespective of the magnitude of $\beta$, if the cost share of the time-deciding agent $A$ is small enough.

As far as the investment threshold is concerned, one can see that, as expected, $y_{N}\left(\psi_{N}\right)$ is increasing in $\eta_{B}{ }^{21}$ and that in the special case where $\eta_{B}=\xi$ we have exactly $y_{N}\left(\psi_{N}\right)=y^{V I}$. In general, when the bargaining power of $B$ is smaller (larger) than the exogenously given $\xi$, the investment takes place inefficiently early (late). Finally, one can check that $\frac{\partial \frac{y_{N}\left(\psi_{N}\right)}{y^{V I}}}{\partial \beta} \gtrless 0$ for $\xi \gtrless \eta_{B}$ which means that when the bargaining power of $B$ is sufficiently low (high), an increase in volatility results in a lower (higher) investment threshold $y_{N}\left(\psi_{N}\right)$ relative to $y^{V I}$.

Given $y_{N}\left(\psi_{N}\right)$ and $\psi_{N}$, we can compute the value of the option to invest both for the potential investor and for the investment partner. For $A$ we obtain

$$
F_{A N}(y)=(1-\xi)\left(\frac{\beta-\eta_{B}}{\beta-\xi}\right)^{\beta} F_{A}^{V I}(y)
$$

and for $B$ we have

$$
F_{B N}(y)=\eta_{B}\left(\frac{\beta-\eta_{B}}{\beta-\xi}\right)^{\beta-1} F_{A}^{V I}(y) .{ }^{22}
$$

Of course the two agents will choose the Nash bargaining solution over the non-cooperative one only if $F_{A N}(y) \geq F_{A}^{V C}(y)$ and $F_{B N}(y) \geq F_{B}^{V C}(y)$ or, alternatively, if $(1-\xi)\left(\frac{\beta-\eta_{B}}{\beta-\xi}\right)^{\beta} \geq\left(\frac{\beta-1}{\beta-1+\xi}\right)^{\beta-1}$ and $\eta_{B}\left(\frac{\beta-\eta_{B}}{\beta-\xi}\right)^{\beta-1} \geq \xi\left(\frac{\beta-1}{\beta-1+\xi}\right)^{\beta}$ hold simultaneously.

\footnotetext{
${ }^{19} \mathrm{It}$ is assumed that the distribution of bargaining power is exogenous and that $\eta_{A}+\eta_{B}=1$ with $\eta_{A} \geq 0$ and $\eta_{B} \geq 0$, where $\eta_{i}$ is the bargaining power of agent $i$ with $i \in\{A, B\}$.

${ }^{20}$ One can also check that $\psi_{N}$ is increasing and convex in $\xi$.

${ }^{21}$ One can also check that $y_{N}\left(\psi_{N}\right)$ is linearly decreasing in $\xi$.

${ }^{22}$ Note that, as expected, $\frac{\partial F_{i N}\left(y_{t}\right)}{\partial \eta_{i}}>0, i \in\{A, B\}$.
} 
It is interesting to see that the condition $F_{A N}(y) \geq F_{A}^{V C}(y)$ implies $\xi>\eta_{B}$ which basically means that the Nash bargaining solution guarantees that the investment will take place (inefficiently) early: $y_{N}\left(\psi_{N}\right)<y^{V I}$. Let's recall that when we solved the same problem under the non-cooperative framework in Section 3.4, we found that the investment would take place inefficiently late: $y^{V C}\left(\psi^{V C}\right)>y^{V I}$. Comparing these two results we derive a quite straightforward conclusion: the adopted game-theoretic framework determines the nature of the interaction between $A$ and $B$ as well as the way that this is reflected on the chosen investment threshold and the value of the option to invest. The importance of this statement will become clearer in the next section where the three-agent case is discussed. Summing up our results:

Proposition 9 In the case where the input is produced in-house and the completion of the project depends on external funding, the Nash bargaining solution can replace the non-cooperative one when the participation conditions $F_{A N}(y) \geq F_{A}^{V C}(y) \Rightarrow(1-\xi)\left(\frac{\beta-\eta_{B}}{\beta-\xi}\right)^{\beta} \geq\left(\frac{\beta-1}{\beta-1+\xi}\right)^{\beta-1}$ and $F_{B N}(y) \geq$ $F_{B}^{V C}(y) \Rightarrow \eta_{B}\left(\frac{\beta-\eta_{B}}{\beta-\xi}\right)^{\beta-1} \geq \xi\left(\frac{\beta-1}{\beta-1+\xi}\right)^{\beta}$ are satisfied. If this is the case, then the investment occurs when the scale parameter reaches a threshold $y_{N}\left(\psi_{N}\right)=\frac{\beta-\xi}{\beta-\eta_{B}} y^{V I}$. Notably, $F_{A N}(y) \geq$ $F_{A}^{V C}(y) \Rightarrow \xi>\eta_{B} \Rightarrow y_{N}\left(\psi_{N}\right)<y^{V I}$ which means that a Nash bargaining solution guarantees that the investment will take place inefficiently early.

For instance, for $\alpha=0.025, \sigma=0.2, r=0.05, y_{0}=\pi_{M}=1, \xi=0.5$ and $I=24$, the Nash bargaining solution can take place for any $\eta_{B} \in[0.147,0.236]$. If e.g. $\eta_{B}=20 \%$, we have $y_{N}=$ $1.45, F_{A N}=15.13$ and $F_{B N}=4,61$. Comparing these with the corresponding values of Table 1 we see that, as expected, the option values of both parties are appreciated and that the investment threshold under Nash bargaining is smaller than the investment threshold under vertical integration.

\subsection{The three-agent case}

Let's now see what is different if a third agent is involved in the completion of the project. Similarly to the presentation of Section 4 it is assumed that the input is produced by an external supplier with market power. The game evolves in the following way:

1. The upstream firm $C$ decides the input price that maximizes her/his individual option value.

2. Given the price of the input, $A$ and $B$ engage in a Nash bargaining in order to decide the compensation that $A$ will submit to $B$ and finally,

3. A decides what is the optimal investment threshold given the price of the input and the decided compensation.

Moving backwards, we begin by studying the behavior of $A$. The optimal investment threshold in the three-agent case is derived as the solution of the following maximization problem:

$$
F_{A 3 N}(y)=\max _{y_{3 N}(\psi, p)}\left((1-\psi) \frac{y_{3 N}(\psi, p) \pi_{M}}{r-a}-(1-\xi) p\right)\left(\frac{y}{y_{3 N}(\psi, p)}\right)^{\beta}
$$

From the first-order condition we obtain

$$
y_{3 N}(\psi, p)=\frac{\beta}{\beta-1} \frac{r-a}{\pi_{M}} p \frac{1-\xi}{1-\psi} .
$$

Given $y_{3 N}(\psi, p), A$ and $B$ engage in a Nash bargaining in order to commonly decide the optimal compensation $\psi_{3 N}$ which is derived as the solution of

$$
\max _{\psi_{3 N}} \frac{\left(\psi_{3 N} \frac{y_{3 N}\left(\psi_{3 N}\right) \pi_{M}}{r-a}-\xi I\right)^{\eta_{B}}}{\left(\left(1-\psi_{3 N}\right) \frac{y_{3 N}\left(\psi_{3 N}\right) \pi_{M}}{r-a}-(1-\xi) I\right)^{\eta_{B}-1}}\left(\frac{y}{y_{3 N}\left(\psi_{3 N}\right)}\right)^{\beta} .
$$


Solving we obtain

$$
\psi_{3 N}=\frac{\xi(\beta-1)+\eta_{B}(1-\xi)}{\beta-\xi} .
$$

Unsurprisingly, we find $\psi_{3 N}=\psi_{N}$. The intuition behind this result is that, as in the non-cooperative case, the presence/absence of $C$ does not affect the interaction between $A$ and $B$ since the exogenously given cost share $\xi$ has to do with the generic investment cost no matter if that is $I$ or $p$.

Finally, the input supplier $C$ observes how $A$ and $B$ behave and chooses the familiar $p_{3 N}=\frac{\beta}{\beta-1} I$ solving $F_{C 3 N}(y)=\max _{p_{3 N}}\left(p_{3 N}-I\right)\left(\frac{y}{y_{3 N}\left(\psi_{3 N}, p_{3 N}\right)}\right)^{\beta}$. Apparently, the price that maximizes the value of the option to invest for the upstream firm is not affected by the distribution of bargaining power between $A$ and $B$. Again, $C$ is indifferent to the means that $A$ uses to fund her $/$ his project.

Now, substituting $\psi_{3 N}$ and $p_{3 N}$ in the formula for the investment threshold we obtain

$$
y_{3 N}\left(\psi_{3 N}, p_{3 N}\right)=\frac{\beta}{\beta-1} \frac{\beta-\xi}{\beta-\eta_{B}} y^{V I}>y_{N}\left(\psi_{N}\right) .
$$

Similarly to $y_{N}\left(\psi_{N}\right)$, the threshold $y_{3 N}\left(\psi_{3 N}, p_{3 N}\right)$ is increasing in $\eta_{B} \cdot{ }^{23}$ One can also check that, contrary to the two-agent case where we had $\frac{\partial \frac{y_{N}\left(\psi_{N}\right)}{y^{V I}}}{\partial \beta} \gtrless 0$ for $\xi \gtrless \eta_{B}$, here we have $\frac{\partial \frac{y_{3 N}\left(\psi_{3 N}, p_{3 N}\right)}{y^{V I}}}{\partial \beta}<0$ which means that as the volatility of the scale parameter increases, the relative investment threshold gets larger, irrespective of how $\eta_{B}$ compares to $\xi$. This has to do with the fact that the delivery price $p_{3 N}$, contrary to $I$, is increasing in the volatility of the scale parameter and, as a result, there is no level of bargaining power low enough to guarantee a negative relationship between the relative investment threshold and the volatility. As a consequence, we have $y_{3 N}\left(\psi_{3 N}, p_{3 N}\right)>y^{V I}$. Note that this is the result of two opposing forces. On one hand, the interaction between $A$ and $B$ drives the investment threshold below $y^{V I}$ but, ${ }^{24}$ on the other, the effect of the presence of $C$ has the opposite direction. ${ }^{25}$ Apparently the second one prevails. It is interesting to recall here that when we discussed the three-agent case under the non-cooperative setting in Section 4, we similarly had $y_{3}\left(\psi_{3}, p_{3}\right)>y^{V I}$. However, there the two effects were not opposing but, on the contrary, they were complementing each other. As a result, the importance of explicitly taking $C$ into account was, to some extent, less obvious.

We conclude with the value of the option to invest for the three agents and we have

$$
\begin{aligned}
& F_{A 3 N}(y)=(1-\xi)\left(\frac{\beta-1}{\beta}\right)^{\beta-1}\left(\frac{\beta-\eta_{B}}{\beta-\xi}\right)^{\beta} F_{A}^{V I}(y), \\
& F_{B 3 N}(y)=\eta_{B}\left(\frac{\beta-1}{\beta}\right)^{\beta-1}\left(\frac{\beta-\eta_{B}}{\beta-\xi}\right)^{\beta-1} F_{A}^{V I}(y), \\
& F_{C 3 N}(y)=\left(\frac{\beta-1}{\beta}\right)^{\beta}\left(\frac{\beta-\eta_{B}}{\beta-\xi}\right)^{\beta} F_{A}^{V I}(y) .
\end{aligned}
$$

$A$ and $B$ will choose the Nash bargaining solution over the non-cooperative one only if $F_{A 3 N}(y) \geq$ $F_{A 3}(y)$ and $F_{B 3 N}(y) \geq F_{B 3}(y)$. As in the previous section, we find that if the conditions $(1-\xi)\left(\frac{\beta-\eta_{B}}{\beta-\xi}\right)^{\beta} \geq$ $\left(\frac{\beta-1}{\beta-1+\xi}\right)^{\beta-1}$ and $\eta_{B}\left(\frac{\beta-\eta_{B}}{\beta-\xi}\right)^{\beta-1} \geq \xi\left(\frac{\beta-1}{\beta-1+\xi}\right)^{\beta}$ hold simultaneously, then both $A$ and $B$ are better

\footnotetext{
${ }^{23}$ Note that, similarly to $y_{N}\left(\psi_{N}\right), y_{3 N}\left(\psi_{3 N}, p_{3 N}\right)$ is linearly decreasing in $\xi$.

${ }^{24}$ This first effect was discussed in Section 5.1.

${ }^{25}$ This second effect was discussed in Section 3.3.
} 
off. Once again this has to do with the fact that the presence/absence of $C$ does not affect the interaction between $A$ and $B$. Finally, as far as $C$ is concerned, the Nash bargaining solution is preferred to the non-cooperative one when $F_{C 3 N}(y) \geq F_{C 3}(y) \Rightarrow\left(\frac{\beta-\eta_{B}}{\beta-\xi}\right)^{\beta}>\left(\frac{\beta-1}{\beta-1+\xi}\right)^{\beta}$. One can easily check that this is always the case since $F_{A 3 N}(y) \geq F_{A 3}(y)$ implies $F_{C 3 N}(y) \geq F_{C 3}(y)$. Concluding we have:

Proposition 10 In the case where the input is outsourced and the completion of the project depends on external funding, the Nash bargaining solution can replace the non-cooperative one when the participation conditions $F_{A 3 N}(y) \geq F_{A 3}(y) \Leftrightarrow F_{A N}(y) \geq F_{A}^{V C}(y)$ and $F_{B 3 N}(y) \geq F_{B 3}(y) \Leftrightarrow$ $F_{B N}(y) \geq F_{B}^{V C}(y)$ hold simultaneously. If this is the case, the investment occurs when the scale parameter reaches a threshold $y_{3 N}\left(\psi_{3 N}, p_{3 N}\right)=\frac{\beta}{\beta-1} \frac{\beta-\xi}{\beta-\eta_{B}} y^{V I}>y^{V I}$ i.e. the investment takes place inefficiently late. Despite the fact that the presence of $B$ favors the acceleration of the project ${ }^{26}$ the presence of $C$, which dictates the postponement of the investment, prevails.

For instance, for $\alpha=0.025, \sigma=0.2, r=0.05, y_{0}=\pi_{M}=1, \xi=0.5$ and $I=24$, the Nash bargaining solution can take place, as before, for any $\eta_{B} \in[0.147,0.236]$. If e.g. $\eta_{B}=20 \%$, we have: $y_{3 N}=4.59, F_{A 3 N}=8.89, F_{B 3 N}=2,71$ and $F_{C 3 N}=5,61$. Comparing these with the corresponding values of Table 1 we see that, as expected, the option values of all three parties are appreciated and that the investment threshold under Nash bargaining is larger than the investment threshold under vertical integration.

\section{Epilogue}

In this paper we consider the investment problem of a firm who contemplates entering an uncertain new market under two conditions. On one hand, an upstream firm with market power is responsible for the provision of a discrete input that is a prerequisite for the completion of the project and, on the other, an exogenously given share of the sunk investment cost is undertaken by an investment partner who claims a share of the project as compensation in return.

Following the real option approach, we build a stochastic dynamic programming model in order to study the interaction among these three agents and we find the following. Firstly, we verify that the optimal investment timing and, consequently, the maximum value of the option to invest are reached when the potential investor acts autonomously (vertically integrated case). On the contrary, the presence of any additional agent involved in the completion of the project causes the delay of the investment which, as we show, also implies a smaller value of the option to invest. However, despite the fact that the presence of any additional agent affects the timing and the value of the option to invest the same way, the magnitude of the effect itself is not the same. Actually, a comparison between external funding and input outsourcing denotes that the former is always preferred to the latter.

Secondly, we focus on the three-agent case and we find that the synchronous involvement of an upstream supplier and an investment partner in the project, constitutes the worst-case scenario since we basically deal with a combination of the corresponding distorting effects. In this case, the value of the option to invest reaches its minimum whereas the investment threshold reaches its maximum.

In the last part of the paper we present the conditions under which it is possible to replace the original non-cooperative setting with a Nash bargaining solution and we show that, even in

\footnotetext{
${ }^{26}$ See Proposition 9.
} 
that case, the optimal investment threshold is unattainable. Actually, our analysis shows that if the upstream firm is absent (i.e. the input is produced in-house) the project realizes inefficiently early whereas, if the upstream firm is present (i.e. the input is outsourced) it realizes inefficiently late. This is, again, evidence of the importance of the nature of the sunk investment cost when modelling investment projects characterized by uncertainty and irreversibility, especially if instead of a single potential investor, an investment partner is also involved in their completion. 


\section{A Appendix}

\section{A.1 The input is produced in-house and the investment is partly externally funded: A review of Section 3.4}

In the main body of the paper we describe the interaction between a potential investor $A$ and an investment partner $B$ following the presentation by Lukas and Welling (2014) according to which $A$ is the game-leader submitting the compensation offer and $B$ is the game-follower choosing the investment timing. This framework seems suitable to describe the efforts of a potential investor who seeks out funding for the business plan that is under consideration. However, one can also consider the case where $B$ is the game-leader submitting the compensation offer and $A$ is the game-follower deciding the investment timing. This framework seems more appropriate to describe partnerships in which a venture capitalist makes the first step declaring her/his interest to invest in an emerging firm. ${ }^{27}$

Moving backwards, we find that the solution of $A$ 's decision problem gives an investment threshold $y^{R V C}=\frac{1-\xi}{1-\psi} y^{V I}$ whereas the solution of $B$ 's decision problem gives a compensation share $\psi^{R V C}=\frac{1-2 \xi+\xi \beta}{\beta-\xi} \cdot{ }^{28}$ Combining the two we find that the optimal investment threshold is given by

$$
y^{R V C}\left(\psi^{R V C}\right)=\frac{\beta-\xi}{\beta-1} y^{V I}\left(>y^{V I}\right) .
$$

As far as the value of the option to invest is concerned, we find that for the potential investor $A$ we have

$$
F_{A}^{R V C}(y)=(1-\xi)\left(\frac{\beta-1}{\beta-\xi}\right)^{\beta} F_{A}^{V I}(y)
$$

whereas for the investment partner $B$ we obtain

$$
F_{B}^{R V C}(y)=\left(\frac{\beta-1}{\beta-\xi}\right)^{\beta-1} F_{A}^{V I}(y) .
$$

One can easily check that, similarly to what we find in Section 3.4, the presence of firm $B$ causes the postponement of the project ${ }^{29}$, making the potential investor worse off with respect to the vertically integrated case.

\section{A.2 The three-agent case: A review of Section 4}

In this section we review the three-agent case as presented in Section 4 assuming however that $A$ and $B$ swap places. More precisely:

1. $C$ is still the game-leader who decides the input price.

2. Given the price of the input, $B$ decides what is the optimal compensation that $\mathrm{s} /$ he should ask from $A$ and

3. A decides what is the optimal investment threshold given the price of the input and the compensation share.

Solving backwards, from the potential investor's maximization problem we obtain $y_{3 R}(\psi, p)=$ $\frac{\beta}{\beta-1} \frac{1-\xi}{1-\psi} \frac{r-a}{\pi_{M}} p$. Taking this into consideration, the investment partner is choosing $\psi_{3 R}=\frac{1-2 \xi+\xi \beta}{\beta-\xi}$

\footnotetext{
${ }^{27}$ Cvitanić et al. (2011) present a similar case.

${ }^{28}$ Check that $\psi^{R V C}$, similarly to $\psi^{V C}$, is increasing in $\xi$, but contrary to $\psi^{V C}$ is decreasing in $\beta$.

${ }^{29}$ Note that $y^{R V C}$, similarly to $y^{V C}$, is decreasing in $\beta$, but contrary to $y^{V C}$ is decreasing in $\xi$.
} 
$\left(=\psi^{R V C}\right) \cdot{ }^{30}$ Finally, the upstream firm, keeping in mind the reactions of $A$ and $B$, decides the optimal price of the input $p_{3 R}=\frac{\beta}{\beta-1} I$. Plugging the compensation offer and the input price in the formula for the investment threshold we obtain

$$
y_{3 R}\left(\psi_{3 R}, p_{3 R}\right)=\frac{\beta}{\beta-1} \frac{\beta-\xi}{\beta-1} y^{V I}\left(>y^{V I}\right) .
$$

Given this, we also have

$$
\begin{aligned}
& F_{A 3 R}(y)=(1-\xi)\left(\frac{\beta-1}{\beta}\right)^{\beta-1}\left(\frac{\beta-1}{\beta-\xi}\right)^{\beta} F_{A}^{V I}(y), \\
& F_{B 3 R}(y)=\left(\frac{\beta-1}{\beta}\right)^{\beta-1}\left(\frac{\beta-1}{\beta-\xi}\right)^{\beta-1} F_{A}^{V I}(y), \\
& F_{C 3 R}(y)=\left(\frac{\beta-1}{\beta}\right)^{\beta}\left(\frac{\beta-1}{\beta-\xi}\right)^{\beta} F_{A}^{V I}(y) .
\end{aligned}
$$

Similarly to Section 4, we find that the synchronous presence of $B$ and $C$ causes the postponement of the project and that this is also reflected on $A$ 's option value.

Finally, note that from Eq. (A.2), Eq. (A.3) and Eq. (A.5) we find that $F^{R V C}(y)=$ $\frac{2 \beta-\beta \xi-1}{\beta-1}\left(\frac{\beta-1}{\beta-\xi}\right)^{\beta} F_{A}^{V I}(y)$ and $F_{3 R}(y)=\left(1-\xi+\frac{\beta-\xi}{\beta-1}+\frac{\beta-1}{\beta}\right)\left(\frac{\beta-1}{\beta}\right)^{\beta-1}\left(\frac{\beta-1}{\beta-\xi}\right)^{\beta} F_{A}^{V I}(y) .{ }^{31}$ Summing up

Proposition 11 A comparison among the option values and the investment triggers derived in Section A.1 and Section A.2 of Appendix A gives the following rankings:

1) $y_{3 R}\left(\psi_{3 R}, p_{3 R}\right)>y^{O S}\left(p^{O S}\right)>y^{R V C}\left(\psi^{R V C}\right)>y^{V I}$,

2) $F_{3 R}(y)<F^{O S}(y)<F^{R V C}(y)<F_{A}^{V I}(y)$,

3) $F_{A 3 R}(y)<F_{A}^{R V C}(y)<F_{A}^{O S}(y)<F_{A}^{V I}(y)$,

4) $F_{B 3 R}(y)<F_{B}^{R V C}(y)$ and

5) $F_{C 3 R}(y)<F_{C}^{O S}(y)$.

Comparing Proposition 11 (where $B$ is the game-leader and $A$ is the game-follower) with Proposition 8 (where $A$ is the game-leader and $B$ is the game-follower) we find that, in both cases, as the number of agents involved in an investment project increases, the completion of the project is postponed at the expense of the project's option value both in the firm and in the industry level. We also find that the rankings of the investment thresholds and the aggregate option values remain the same whereas the only difference that we observe has to do with the ranking of $A$ 's option values. As one can see, when $A$ is the game-follower (game-leader), an interaction with $C(B)$ is preferred to an interaction with $B(C)$ which means that the way that $A$ is affected by the presence of the alien firms depends on the role that $\mathrm{s} /$ he has in the game.

Another interesting point is that $F_{A}^{R V C}(y)<F_{A}^{V C}(y)$ and $F_{B}^{R V C}(y)>F_{B}^{V C}(y)$ which means that being the game-leader is always preferable, no matter the values of $\xi$ and $\beta$. Finally, one can also check that $y^{R V C}\left(\psi^{R V C}\right) \gtrless y^{V C}\left(\psi^{V C}\right)$ and $y_{3 R}\left(\psi_{3 R}, p_{3 R}\right) \gtrless y_{3}\left(\psi_{3}, p_{3}\right)$, and consequently that $F^{R V C}(y) \lessgtr F^{V C}(y)$ and $F_{3 R}(y) \lessgtr F_{3}(y)$, when $0.5 \gtrless \xi$. In words, it is socially optimal for the agent who undertakes the lion's share of the sunk investment cost to be the game-leader either when the input is outsourced or not. This means that the analysis of the main body of the paper where $A$ is the game-leader and $B$ is the game-follower would be preferred from a social point of view for $0.5<\xi$ whereas the analysis presented here would be socially preferable for $0.5>\xi$.

\footnotetext{
${ }^{30}$ Check that the equality $\psi_{3 R}=\psi^{R V C}$ is analogous to the equality $\psi_{3}=\psi^{V C}$ that we find in Section 4 of the main body of the paper.

${ }^{31}$ We define $F^{R V C}(y) \equiv F_{A}^{R V C}(y)+F_{B}^{R V C}(y)$ and $F_{3 R}(y) \equiv F_{A 3 R}(y)+F_{B 3 R}(y)+F_{C 3 R}(y)$.
} 


\section{B The compensation as the product of Nash bargaining}

\section{B.1 The input is produced in-house and the investment is partly externally funded: A review of Section 5.1}

In Section A.1 of Appendix A we presented a leader-follower game where $B$ decides the compensation and, given that, $A$ chooses the optimal investment threshold. Let's now see what is different if the compensation is the product of bargaining between the two agents. The game evolves in the following way: initially $A$ and $B$ bargain over the compensation share $\psi$ and then, given that, $B$ decides the optimal investment threshold. Notice that contrary to Section A.1, the time-deciding agent is $B$, not $A$. Alternatively put, our goal in this section is to find the conditions under which $A$ would be willing to let $B$ decide the timing of the investment, given that the compensation share will be the product of bargaining between the two agents instead of a unilateral decision of $B$.

Starting with the maximization problem of the time-deciding agent $B$ we obtain $y_{N R}=\frac{\xi}{\psi} y^{V I}$. Moving one step back, the two parties bargain anticipating that $B$ will invest as soon as $y_{t}$ reaches the threshold $y_{N R}$. Given this, the bargaining over the compensation share gives $\psi_{N R}=\xi \frac{\beta-1+\eta_{B}}{\beta-1+\xi}$ 32 and, consequently,

$$
y_{N R}\left(\psi_{N R}\right)=\frac{\beta-1+\xi}{\beta-1+\eta_{B}} y^{V I} \cdot 33
$$

Given the compensation offer and the optimal investment threshold, we can compute the value of the option to invest both for the potential investor and for the time-deciding investment partner. More precisely, for $A$ we obtain

$$
F_{A N R}(y)=\left(1-\eta_{B}\right)\left(\frac{\beta-1+\eta_{B}}{\beta-1+\xi}\right)^{\beta-1} F_{A}^{V I}(y),
$$

and for $B$ we have

$$
F_{B N R}(y)=\xi\left(\frac{\beta-1+\eta_{B}}{\beta-1+\xi}\right)^{\beta} F_{A}^{V I}(y)
$$

As expected, $A$ and $B$ will choose the Nash bargaining solution over the non-cooperative one only if $F_{A N R}(y) \geq F_{A}^{R V C}(y)$ and $F_{B N R}(y) \geq F_{B}^{R V C}(y)$ or, alternatively, if $\left(1-\eta_{B}\right)\left(\frac{\beta-1+\eta_{B}}{\beta-1+\xi}\right)^{\beta-1} \geq$ $(1-\xi)\left(\frac{\beta-1}{\beta-\xi}\right)^{\beta}$ and $\xi\left(\frac{\beta-1+\eta_{B}}{\beta-1+\xi}\right)^{\beta} \geq\left(\frac{\beta-1}{\beta-\xi}\right)^{\beta-1}$ hold simultaneously. One can also easily check that the condition $F_{B N R}(y) \geq F_{B}^{R V C}(y)$ implies $\xi<\eta_{B}$ which means that any Nash bargaining solution guarantees that the investment will take place (inefficiently) early. Note that this is no different from what we found in Section 5.1 of the main body of the paper.

\section{B.2 The three-agent case: A review of Section 5.2}

Let's now see what is different if the input is outsourced. Our starting point is again the investment threshold decision by $B$. From the first-order condition we have $y_{3 N R}(\psi, p)=\frac{\beta}{\beta-1} \frac{r-a}{\pi_{M}} p \frac{\xi}{\psi}$. Moving one step back, $A$ and $B$ bargain anticipating that $B$ will invest as soon as $y_{t}$ reaches the chosen

\footnotetext{
${ }^{32}$ One can check that the compensation share $\psi_{N R}$ increases linearly in $\eta_{B}$ and that it is also increasing and concave in $\xi$. Finally, $\frac{\partial \psi_{N R}}{\partial \beta} \gtrless 0$ for $\xi \gtrless \eta_{B}$.

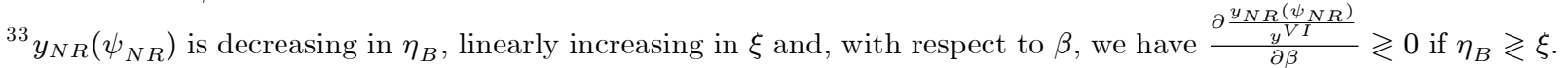
This basically means that in the special case where $\eta_{B}=\xi$ we have exactly $y_{N R}\left(\psi_{N R}\right)=y^{V I}$ but, in general, when the bargaining power of $B$ is sufficiently low (high), an increase in volatility results in a higher (lower) investment threshold $y_{N R}\left(\psi_{N R}\right)$ relative to $y^{V I}$.
} 
threshold and eventually choose $\psi_{3 N R}=\xi \frac{\beta-1+\eta_{B}}{\beta-1+\xi}\left(=\psi_{N R}\right) .{ }^{34}$ Finally, the game-leader $C$ observes the behavior of $A$ and $B$ and decides the input price $p_{3 N R}=\frac{\beta}{\beta-1} I$.

Substituting the optimal price and the compensation offer in the investment threshold from above we have

$$
y_{3 N R}\left(\psi_{3 N R}, p_{3 N R}\right)=\frac{\beta}{\beta-1} \frac{\beta-1+\xi}{\beta-1+\eta_{B}} y^{V I} .
$$

Note that $y_{3 N R}\left(\psi_{3 N R}, p_{3 N R}\right)>y^{V I} .35$ This is the result of two opposing forces. On one hand, the interaction between $A$ and $B$ drives the investment trigger below $y^{V I}$ but, ${ }^{36}$ on the other, the effect of the presence of $C$ has the opposite direction. ${ }^{37}$ Apparently, the second one prevails. It is interesting to recall that Eq. (A.4) that corresponds to the non-cooperative case gives also an investment threshold higher than $y^{V I}: y_{3 R}\left(\psi_{3 R}, p_{3 R}\right)>y^{V I}$. However, there the two effects were not opposing but, on the contrary, they were complementing each other. Note that our analysis here is totally symmetric to the one presented in Section 5.2 of the main body of the paper.

Keeping in mind the formulas for $\psi_{3 N R}, p_{3 N R}$ and $y_{3 N R}\left(\psi_{3 N R}, p_{3 N R}\right)$, the option values for the three agents are

$$
\begin{aligned}
& F_{A 3 N R}(y)=\left(1-\eta_{B}\right)\left(\frac{\beta-1}{\beta} \frac{\beta-1+\eta_{B}}{\beta-1+\xi}\right)^{\beta-1} F_{A}^{V I}(y), \\
& F_{B 3 N R}(y)=\xi\left(\frac{\beta-1}{\beta}\right)^{\beta-1}\left(\frac{\beta-1+\eta_{B}}{\beta-1+\xi}\right)^{\beta} F_{A}^{V I}(y), \\
& F_{C 3 N R}(y)=\left(\frac{\beta-1}{\beta}\right)^{\beta}\left(\frac{\beta-1+\eta_{B}}{\beta-1+\xi}\right)^{\beta} F_{A}^{V I}(y) .
\end{aligned}
$$

$A$ and $B$ will choose the Nash bargaining solution over the non-cooperative one only if $F_{A 3 N R}(y) \geq$ $F_{A 3 R}(y)$ and $F_{B 3 N R}(y) \geq F_{B 3 R}(y)$ or, alternatively, if the familiar $\left(1-\eta_{B}\right)\left(\frac{\beta-1+\eta_{B}}{\beta-1+\xi}\right)^{\beta-1} \geq$ $(1-\xi)\left(\frac{\beta-1}{\beta-\xi}\right)^{\beta}$ and $\xi\left(\frac{\beta-1+\eta_{B}}{\beta-1+\xi}\right)^{\beta} \geq\left(\frac{\beta-1}{\beta-\xi}\right)^{\beta-1}$ hold simultaneously. As far as $C$ is concerned, the Nash bargaining solution is preferred to the non-cooperative one when $F_{C 3 N R}(y) \geq F_{C 3 R}(y) \Rightarrow$ $\left(\frac{\beta-1+\eta_{B}}{\beta-1+\xi}\right)^{\beta} \geq\left(\frac{\beta-1}{\beta-\xi}\right)^{\beta}$. One can easily see that this is always the case since $F_{B 3 N R}(y) \geq F_{B 3 R}(y)$ implies $F_{C 3 N R}(y) \geq F_{C 3 R}(y)$. Concluding we have:

Proposition 12 In the case where the input is outsourced and the completion of the project depends on external funding, the Nash bargaining solution ${ }^{38}$ can replace the non-cooperative one $e^{39}$ when the participation conditions $F_{A 3 N R}(y) \geq F_{A 3 R}(y) \Leftrightarrow F_{A N R}(y) \geq F_{A}^{R V C}(y)$ and $F_{B 3 N R}(y) \geq F_{B 3 R}(y) \Leftrightarrow$ $F_{B N R}(y) \geq F_{B}^{R V C}(y)$ hold simultaneously. If this is the case, the investment occurs when the scale parameter reaches a threshold $y_{3 N R}\left(\psi_{3 N R}, p_{3 N R}\right)=\frac{\beta}{\beta-1} \frac{\beta-1+\xi}{\beta-1+\eta_{B}} y^{V I}>y^{V I}$ i.e. the investment takes place inefficiently late. Despite the fact that the presence of $B$ favors the acceleration of the project ${ }^{40}$ the presence of $C$, which dictates the postponement of the investment, prevails.

\footnotetext{
${ }^{34}$ Check that the equality $\psi_{3 N R}=\psi_{N R}$ is analogous to the equality $\psi_{3 N}=\psi_{N}$ that we find in Section 5.2 of the main body of the paper.

${ }^{35}$ Note also that $\frac{\partial \frac{y_{3 N R}\left(\psi_{3 N R}, p_{3 N R}\right)}{y^{V I}}}{\partial \beta}<0$.

${ }^{36}$ This effect was discussed in Section B.1 of Appendix B.

${ }^{37}$ This effect was discussed in Section 3.3 of the main body of the paper.

${ }^{38}$ Section B.2 of Appendix B.

${ }^{39}$ Section A.2 of Appendix A.

${ }^{40}$ According to Section B.1 of Appendix B.
} 


\section{References}

[1] Alvarez LHR, Stenbacka R. 2007. Partial outsourcing: a real options perspective. International Journal of Industrial Organization 25: 91-102.

[2] Azevedo A, Paxson D. 2014 Developing real option game models, European Journal of Operational Research 237: 909-920.

[3] Banerjee S, Güçbilmez IU, Pawlina G. 2014. Optimal exercise of jointly held real options: a Nash bargaining approach with value diversion. European Journal of Operational Research 239 (2): 565-578.

[4] Benson D, Ziedonis RH. 2009. Corporate venture capital as a window on new technologies: Implications for corporate investors when acquiring startups. Organization Science 20: 352376.

[5] Biehl M, Cook W, Johnston DA. 2006.The efficiency of joint decision making in buyer-supplier relationships. Annals of Operations Research 145:15-34.

[6] Billette de Villemeur E, Ruble R, Versaevel B. 2014. Investment timing and vertical relationships. International Journal of Industrial Organization 33: 110-123.

[7] Chen P. 2012. The investment strategies for a dynamic supply chain under stochastic demands. International Journal of Production Economics 139: 80-89.

[8] Chesbrough H, Schwartz K. 2007. Innovating business models with co-development partnerships. Research Technology Management 50: 55-59.

[9] Chevalier-Roignant B, Flath CM, Huchzermeier A, Trigeorgis L. 2011. Strategic investment under uncertainty: a synthesis. European Journal of Operational Research 215: 639-650.

[10] Cvitanić J, Radas S, Śikić H. 2011. Co-development ventures: optimal time of entry and profit-sharing. Journal of Economic Dynamics and Control 35(10): 1710-1730.

[11] Di Corato L, Moretto M, Rossini G. 2017. Financing flexibility: The case of outsourcing. Journal of Economic Dynamics and Control 76: 35-65.

[12] Dixit A. 1993, The art of smooth pasting, Fundamentals of Pure and Applied Economics 55, Routledge.

[13] Dixit A, Pindyck RS. 1994. Investment under Uncertainty. Princeton University Press, Princeton.

[14] Dushnitsky G, Lenox MJ. 2005a. When do incumbents learn from entrepreneurial ventures? Corporate venture capital and investing firm innovation rates. Research Policy 34: 615-639.

[15] Dushnitsky G, Lenox MJ. 2005b. When do firms undertake R\&D by investing in new ventures? Strategic Management Journal 26(10): 947-965.

[16] Folta TB. 1998. Governance and uncertainty: The trade-off between administrative control and commitment. Strategic Management Journal 19: 1007-1028.

[17] Folta TB, Miller KD. 2002. Real options in equity partnerships. Strategic Management Journal 23: $77-88$. 
[18] Guimarães Dias M, Teixeira J. 2010. Continuous-Time Option Games: Review of Models and Extensions. Multinational Finance Journal 14: 219-254.

[19] Huisman K, Kort PM, Pawlina G, Thijssen J. 2004. Strategic investment under uncertainty: Merging real options with game theory. Zeitschrift fr Betriebswirtschaft 74: 97-123.

[20] Kogut B. 1991. Joint ventures and the option to expand and acquire. Management Science 37: 19-33.

[21] Kogut B, Kulatilaka N. 1994. Operating flexibility, global manufacturing, and the option value of a multinational network. Management Science 40: 123-139.

[22] Lambrecht BM. 2004. The timing and terms of mergers motivated by economies of scale. Journal of Financial Economics 72(1): 41-62.

[23] Li J, Dhanaraj C, Shockley RL. 2008. Joint venture evolution: extending the real options approach. Managerial and Decision Economics 29: 317-336.

[24] Lukas E, Mölls S, Welling A. 2016. Venture capital, staged financing and optimal funding policies under uncertainty. European Journal of Operational Research 250(1): 305-313.

[25] Lukas E, Welling A. 2014. Investment timing and eco(nomic)-efficiency of climate-friendly investments in supply chains. European Journal of Operational Research 233(2) (Special Issue: Eco-Efficient Green Supply Chain Management): 448-457.

[26] McDonald R, Siegel D. 1986. The value of waiting to invest. Quarterly Journal of Economics 101(4): 707-728.

[27] McGrath RG. 1997. A real options logic for initiating technology positioning investments. Academy of Management Review 22: 974-996.

[28] McGrath RG, Nerkar A. 2004. Real options reasoning and a new look at the R\&D investment strategies of pharmaceutical firms. Strategic Management Journal 25: 1-21.

[29] Miller M, Modigliani F. 1961. Dividend policy, growth, and the valuation of shares. The Journal of Business 34(4): 411-433.

[30] Morellec E, Zhdanov A. 2005. The dynamics of mergers and acquisitions. Journal of Financial Economics 77(3): 649-672.

[31] Moretto M, Rossini G. 2012. Flexible outsourcing. Managerial and Decision Economics 33: 47-59.

[32] Mulherin JH, Boone AL. 2000. Comparing acquisitions and divestures. Journal of Corporate Finance 6: 117-139.

[33] Myers SC. 1977. Determinants of corporate borrowing. Journal of Financial Economics 5(2): $147-176$.

[34] O'Brien JP, Folta TB, Johnson DR. 2003. A real options perspective on entrepreneurial entry in the face of uncertainty. Managerial and Decision Economics 24: 515-533.

[35] Puranam P, Singh H, Chaudhuri S. 2009. Integrating acquired capabilities: when structural integration is (un)necessary. Organization Science 20(2): 313-328. 
[36] Quinn JB. 2000. Outsourcing innovation: the new engine of growth. Sloan Management Review 41: $13-28$.

[37] Reuer JJ, Tong TW. 2007. Corporate investments and growth options. Managerial and Decision Economics 28: 863-877.

[38] Roberts EB, Berry CA. 1985. Entering new business: selecting strategies for success. Sloan Management Review 26(2): 57-71.

[39] Teixeira JCA. 2014. Outsourcing with debt financing. Portoguese Economic Journal 13: 1-24.

[40] Tirole J, 1988. The Theory of Industrial Organization. MIT Press, Cambridge.

[41] Tong TW, Li Y. 2011. Real options and investment mode: evidence from corporate venture capital and acquisition. Organization Science 22(3): 659-674.

[42] Triantis A, Hodder J. 1990. Valuing flexibility as a complex option. The Journal of Finance 45(2): 549-565.

[43] Vrande van de VJA, Vanhaverbeke W. 2013. How prior corporate venture capital investments shape technological alliances: a real options approach. Entrepreneurship Theory and Practice 37 (5): 1019-1043.

[44] Zhou Y-W, Yang S. 2008. Pricing coordination in supply chains through revenue sharing contracts. Information and Management Sciences 19 (1): 31-51. 